\title{
Argument estimates for certain analytic functions
}

\author{
By Mamoru Nunokawa, ${ }^{*)}$ Shigeyoshi Owa, $\left.{ }^{* *}\right)$ Hitoshi SAitoh, ${ }^{* * *}$ \\ and Nicolae N. PASCU ${ }^{* * * *)}$ \\ (Communicated by Heisuke HironakA, M. J. A., Dec. 12, 2003)
}

Abstract: Let $p(z)$ be analytic in the open unit disk $\mathbf{U}$ with $p(0)=1$ and $p^{\prime}(0)=0$. S. S. Miller and P. T. Mocanu (J. Math. Anal. Appl. 276 (2002)) have shown some interesting subordination theorems for such functions $p(z)$. The object of the present paper is to discuss some sufficient conditions for arguments of $p(z)$ to be $|\arg p(z)|<(\pi / 2) \rho$ for $z \in \mathbf{U}$.

Key words: Analytic function; argument estimate; subordination.

1. Introduction. Let $p(z)$ be analytic in the open unit disk $\mathbf{U}=\{z \in \mathbf{C}:|z|<1\}$ with $p(0)=1$ and $p^{\prime}(0)=0$. For such functions $p(z)$, Miller and Mocanu [3] have shown some interesting subordination theorems.

Theorem A $([3])$. For $1 / 2<\rho \leqq 1$ define the function $q(z)$ by

$$
q(z)=q_{\rho}(z)=\left(\frac{1+z}{1-z}\right)^{\rho}
$$

and let $t_{0} \in(0,1)$ be the unique solution of

$$
\begin{aligned}
t^{\rho}\{( & 1-\rho) t^{2} \cos \left(\frac{\pi}{2} \rho\right)+t \sin \left(\frac{\pi}{2} \rho\right) \\
& \left.-(1-\rho) \cos \left(\frac{\pi}{2} \rho\right)\right\}+t^{2}-1=0 .
\end{aligned}
$$

If $p(z)$ is analytic in $\mathbf{U}$, with $p(0)=1, p^{\prime}(0)=0$ and

$$
\begin{aligned}
& \left|\arg \left(z p^{\prime}(z)+p(z)^{2}+p(z)\right)\right| \\
& \quad<\frac{\pi}{2}(\rho+1)-\operatorname{Tan}^{-1}\left(\frac{t_{0}}{1+\rho-(1-\rho) t_{0}^{2}}\right),
\end{aligned}
$$

then $p(z) \prec q_{\rho}(z)$, where the symbol " $\prec$ " means the subordinations.

To discuss our problems for functions $p(z)$, we need the following lemma due to Hallenbeck and Ruscheweyh [2] which is the same as one by Fukui and Sakaguchi [1].

\footnotetext{
2000 Mathematics Subject Classification. Primary 30C45.

*) Emeritus Professor, Department of Mathematics, University of Gunma, 4-2, Aramaki, Maebashi, Gunma 3718510 .

**) Department of Mathematics, Kinki University, 3-4-1, Kowakae, Higashi-Osaka, Osaka 577-8502.

***) Department of Mathematics, Gunma National College of Technology, 580, Toriba, Maebashi, Gunma 371-8530.

****) Department of Mathematics, Transilvania University of Brasov, R-2200 Brasov, Romania.
}

Lemma 1.1. Let $p(z)$ be analytic in $|z|<R$ and $p^{(k)}(0)=0(0 \leqq k \leqq n)$. Then if $|p(z)|$ attains its maximum value on the circle $|z|=r<R$ at $a$ point $z_{0}$, we have

$$
\frac{z_{0} p^{\prime}\left(z_{0}\right)}{p\left(z_{0}\right)} \geqq n+1
$$

Applying the above lemma, we derive

Lemma 1.2. Let $p(z)$ be analytic in $\mathbf{U}, p(0)=$ $1, p^{\prime}(0)=0$, and let $p(z) \neq 0(z \in \mathbf{U})$. If there exists a point $z_{0} \in \mathbf{U}$ such that

$$
|\arg p(z)|<\frac{\pi}{2} \alpha \quad\left(|z|<\left|z_{0}\right|\right)
$$

and

$$
\left|\arg p\left(z_{0}\right)\right|=\frac{\pi}{2} \alpha
$$

for some $\alpha>0$, then we have

$$
\frac{z_{0} p^{\prime}\left(z_{0}\right)}{p\left(z_{0}\right)}=i \alpha k
$$

where

$$
k \geqq\left(a+\frac{1}{a}\right) \geqq 2 \quad \text { when } \quad \arg p\left(z_{0}\right)=\frac{\pi}{2} \alpha
$$

and

$$
k \leqq-\left(a+\frac{1}{a}\right) \leqq-2 \quad \text { when } \quad \arg p\left(z_{0}\right)=-\frac{\pi}{2} \alpha
$$

where $p\left(z_{0}\right)^{1 / \alpha}= \pm$ ia and $a>0$.

Proof. We use the same manner which was used by Nunokawa [4] for the proof of the lemma. Let us put

$$
q(z)=p(z)^{1 / \alpha}
$$

Then we see that $\operatorname{Re} q(z)>0\left(|z|<\left|z_{0}\right|\right), \operatorname{Re} q\left(z_{0}\right)=$ $0, q(0)=1$ and $q^{\prime}(0)=0$. Defining the function $\phi(z)$ 
by

$$
\phi(z)=\frac{1-q(z)}{1+q(z)}
$$

we have that $\phi(0)=0,|\phi(z)|<1\left(|z|<\left|z_{0}\right|\right)$, and $\left|\phi\left(z_{0}\right)\right|=1$.

In view of Lemma 1.1, we know that

$$
\begin{aligned}
\frac{z_{0} \phi^{\prime}\left(z_{0}\right)}{\phi\left(z_{0}\right)} & =\frac{-2 z_{0} q^{\prime}\left(z_{0}\right)}{1-q\left(z_{0}\right)^{2}} \\
& =\frac{-2 z_{0} q^{\prime}\left(z_{0}\right)}{1+\left|q\left(z_{0}\right)\right|^{2}} \geqq 2 .
\end{aligned}
$$

It follows from (1.5) that

$$
-z_{0} q^{\prime}\left(z_{0}\right) \geqq\left(1+\left|q\left(z_{0}\right)\right|^{2}\right)
$$

and $z_{0} q^{\prime}\left(z_{0}\right)$ is a negative real number. Since $q\left(z_{0}\right)$ is a non-vanishing pure imaginary number, we can put $q\left(z_{0}\right)=i a$, where $a$ is a non-vanishing real number. We have, for $a>0$,

$$
\begin{aligned}
\operatorname{Im}\left(\frac{z_{0} q^{\prime}\left(z_{0}\right)}{q\left(z_{0}\right)}\right) & =\operatorname{Im}\left(-\frac{i z_{0} q^{\prime}\left(z_{0}\right)}{\left|q\left(z_{0}\right)\right|}\right) \\
& \geqq\left(\frac{1+a^{2}}{a}\right) \geqq 2
\end{aligned}
$$

and, for $a<0$,

$$
\begin{aligned}
\operatorname{Im}\left(\frac{z_{0} q^{\prime}\left(z_{0}\right)}{q\left(z_{0}\right)}\right) & =\operatorname{Im}\left(\frac{i z_{0} q^{\prime}\left(z_{0}\right)}{\left|q\left(z_{0}\right)\right|}\right) \\
& \leqq-\left(\frac{1+a^{2}}{a}\right) \leqq-2 .
\end{aligned}
$$

On the other hand, it follows that

$$
\frac{z_{0} q^{\prime}\left(z_{0}\right)}{q\left(z_{0}\right)}=\frac{1}{\alpha}\left(\frac{z_{0} p^{\prime}\left(z_{0}\right)}{p\left(z_{0}\right)}\right) .
$$

This completes the proof of Lemma 1.2.

2. Argument estimates. Our first property for argument estimates of analytic function $p(z)$ is contained in

Theorem 2.1. Let $p(z)$ be analytic in $\mathbf{U}$ with $p(0)=1$ and $p^{\prime}(0)=0$. If $p(z)$ satisfies

(2.1) $\left|\arg \left(z p^{\prime}(z)+p(z)^{2}+\alpha p(z)\right)\right|<\pi \rho \quad(z \in \mathbf{U})$ for some $\alpha(\alpha>0), \rho\left(0<\rho \leqq \rho_{0}\right)$, where $\rho_{0}(0<$ $\left.\rho_{0}<1\right)$ is given by

$$
\tan \left(\frac{\pi}{2} \rho_{0}\right)=\frac{2}{\alpha} \rho_{0},
$$

then

$$
|\arg p(z)|<\frac{\pi}{2} \rho \quad(z \in \mathbf{U}) .
$$

Proof. Let a function $p(z)$ satisfy the conditions of the theorem. If there exists a point $z_{0} \in \mathbf{U}$ such that

$$
|\arg p(z)|<\frac{\pi}{2} \rho \quad\left(|z|<\left|z_{0}\right|\right)
$$

and

$$
\left|\arg p\left(z_{0}\right)\right|=\frac{\pi}{2} \rho,
$$

then applying Lemma 1.2, we have that

$$
\frac{z_{0} p^{\prime}\left(z_{0}\right)}{p\left(z_{0}\right)}=i \rho k
$$

where

$$
k \geqq a+\frac{1}{a} \geqq 2 \quad \text { when } \quad \arg p\left(z_{0}\right)=\frac{\pi}{2} \rho
$$

and

$$
k \leqq-\left(a+\frac{1}{a}\right) \leqq-2 \quad \text { when } \quad \arg p\left(z_{0}\right)=-\frac{\pi}{2} \rho
$$

with $p\left(z_{0}\right)^{1 / \rho}= \pm i a(a>0)$. It follows that, for $\arg p\left(z_{0}\right)=\pi / 2 \rho$ and $k \geqq a+1 / a \geqq 2$,

$$
\begin{aligned}
\arg & \left(z_{0} p^{\prime}\left(z_{0}\right)+p\left(z_{0}\right)^{2}+\alpha p\left(z_{0}\right)\right) \\
& =\arg p\left(z_{0}\right)\left(\frac{z_{0} p^{\prime}\left(z_{0}\right)}{p\left(z_{0}\right)}+p\left(z_{0}\right)+\alpha\right) \\
& =\frac{\pi}{2} \rho+\arg \left(i \rho k+a^{\rho} e^{i \frac{\pi}{2} \rho}+\alpha\right) \\
& =\frac{\pi}{2} \rho+\operatorname{Tan}^{-1}\left(\frac{\rho k+a^{\rho} \sin \left(\frac{\pi}{2} \rho\right)}{\alpha+a^{\rho} \cos \left(\frac{\pi}{2} \rho\right)}\right) .
\end{aligned}
$$

Since, by $0<\rho \leqq \rho_{0}<1$ and $k \geqq 2$,

$$
\begin{aligned}
& \operatorname{Tan}^{-1}\left(\frac{\rho k+a^{\rho} \sin \left(\frac{\pi}{2} \rho\right)}{\alpha+a^{\rho} \cos \left(\frac{\pi}{2} \rho\right)}\right) \\
& \geqq \operatorname{Tan}^{-1}\left(\frac{2 \rho+a^{\rho} \sin \left(\frac{\pi}{2} \rho\right)}{\alpha+a^{\rho} \cos \left(\frac{\pi}{2} \rho\right)}\right)>0,
\end{aligned}
$$

we define $g(a)$ by

$$
g(a)=\frac{2 \rho+a^{\rho} \sin \left(\frac{\pi}{2} \rho\right)}{\alpha+a^{\rho} \cos \left(\frac{\pi}{2} \rho\right)} \quad(a>0) .
$$

Noting that

$$
g^{\prime}(a)=\frac{\alpha \rho a^{\rho-1} \cos \left(\frac{\pi}{2} \rho\right)\left(\tan \left(\frac{\pi}{2} \rho\right)-\frac{2 \rho}{\alpha}\right)}{\left(\alpha+a^{\rho} \cos \left(\frac{\pi}{2} \rho\right)\right)^{2}},
$$

we define $h(\rho)$ by

$$
h(\rho)=\tan \left(\frac{\pi}{2} \rho\right)-\frac{2 \rho}{\alpha} \quad\left(0<\rho \leqq \rho_{0}<1\right) .
$$

Then $h(0)=0, h\left(\rho_{0}\right)=0$, and 


$$
h^{\prime \prime}(\rho)=\frac{\pi^{2}}{2} \sec ^{2}\left(\frac{\pi}{2} \rho\right) \tan \left(\frac{\pi}{2} \rho\right)>0 .
$$

This shows that $g^{\prime}(a) \leqq 0$ for $a>0$, that is, that

$$
\begin{aligned}
& \operatorname{Tan}^{-1}\left(\frac{\rho k+a^{\rho} \sin \left(\frac{\pi}{2} \rho\right)}{\alpha+a^{\rho} \cos \left(\frac{\pi}{2} \rho\right)}\right) \\
& \geqq \operatorname{Tan}^{-1}\left(\tan \left(\frac{\pi}{2} \rho\right)\right)=\frac{\pi}{2} \rho .
\end{aligned}
$$

Therefore, we conclude that

$$
\arg \left(z_{0} p^{\prime}\left(z_{0}\right)+p\left(z_{0}\right)^{2}+\alpha p\left(z_{0}\right)\right) \geqq \pi \rho
$$

when $\arg p\left(z_{0}\right)=(\pi / 2) \rho$.

Similarly, for $\arg p\left(z_{0}\right)=-(\pi / 2) \rho$ and $k \leqq$ $-(a+1 / a) \leqq-2$, we have that

$$
\begin{aligned}
(2.12) \quad \arg & \left(z_{0} p^{\prime}\left(z_{0}\right)+p\left(z_{0}\right)^{2}+\alpha p\left(z_{0}\right)\right) \\
= & -\frac{\pi}{2} \rho+\arg \left(i \rho k+a^{\rho} e^{-i \frac{\pi}{2} \rho}+\alpha\right) \\
= & -\frac{\pi}{2} \rho+\operatorname{Tan}^{-1}\left(\frac{\rho k-a^{\rho} \sin \left(\frac{\pi}{2} \rho\right)}{\alpha+a^{\rho} \cos \left(\frac{\pi}{2} \rho\right)}\right) \\
\leqq & -\frac{\pi}{2} \rho+\operatorname{Tan}^{-1}\left(\frac{-2 \rho-a^{\rho} \sin \left(\frac{\pi}{2} \rho\right)}{\alpha+a^{\rho} \cos \left(\frac{\pi}{2} \rho\right)}\right) \\
= & -\frac{\pi}{2} \rho-\operatorname{Tan}^{-1}\left(\frac{2 \rho+a^{\rho} \sin \left(\frac{\pi}{2} \rho\right)}{\alpha+a^{\rho} \cos \left(\frac{\pi}{2} \rho\right)}\right) \\
& \leqq-\frac{\pi}{2} \rho-\frac{\pi}{2} \rho=-\pi \rho .
\end{aligned}
$$

Thus, for such a point $z_{0} \in \mathbf{U}$. we see that

$$
\left|\arg \left(z_{0} p^{\prime}\left(z_{0}\right)+p\left(z_{0}\right)^{2}+\alpha p\left(z_{0}\right)\right)\right| \geqq \pi \rho,
$$

which contradicts our condition for $p(z)$.

Consequently, we conclude that

$$
|\arg p(z)|<\frac{\pi}{2} \rho \quad(z \in \mathbf{U}) .
$$

Example 2.1. Let us consider the function $p(z)$ defined by

$$
p(z)=1+\frac{1}{5} z^{2} .
$$

Then we see that

$$
z p^{\prime}(z)+p(z)^{2}+\frac{1}{2} p(z)=\frac{3}{2}+\frac{9}{10} z^{2}+\frac{1}{25} z^{4} .
$$

Letting $\alpha=1 / 2$ and

$$
\rho=\frac{1}{\pi} \operatorname{Sin}^{-1}\left(\frac{19}{30}\right)
$$

in Theorem 2.1, we have that

$$
\left|\arg \left(z p^{\prime}(z)+p(z)^{2}+\frac{1}{2} p(z)\right)\right|<\pi \rho=\operatorname{Sin}^{-1}\left(\frac{19}{30}\right)
$$

and

$$
|\arg p(z)|<\operatorname{Sin}^{-1}\left(\frac{1}{5}\right)<\frac{\pi}{2} \rho .
$$

If we take $\alpha=1$ in Theorem 2.1, then

Corollary 2.1. Let $p(z)$ be analytic in $\mathbf{U}$ with $p(0)=1$ and $p^{\prime}(0)=0$. If $p(z)$ satisfies

$$
\left|\arg \left(z p^{\prime}(z)+p(z)^{2}+p(z)\right)\right|<\pi \rho \quad(z \in \mathbf{U})
$$

for some $\rho(0<\rho \leqq 1 / 2)$, then

$$
|\arg p(z)|<\frac{\pi}{2} \rho \quad(z \in \mathbf{U}) .
$$

Remark 2.1. (1) If $\alpha=4 / 5$, then $0<\rho \leqq$ $\rho_{0}$ and $0.647873<\rho_{0}<0.647874$.

(2) If $\alpha=1 / 2$, then $0<\rho \leqq \rho_{0}$ and $0.809251<\rho_{0}<0.809252$.

(3) If $\alpha=1 / 3$, then $0<\rho \leqq \rho_{0}$ and $0.880966<\rho_{0}<0.880967$.

(4) If $\alpha=1 / 4$, then $0<\rho \leqq \rho_{0}$ and $0.913417<\rho_{0}<0.913418$.

(5) If $\alpha=1.1$, then $0<\rho \leqq \rho_{0}$ and $0.401248<$ $\rho_{0}<0.401249$.

(6) If $\alpha=1.2$, then $0<\rho \leqq \rho_{0}$ and $0.262943<$ $\rho_{0}<0.262944$.

(7) If $\alpha=1.3$, then there is no $\rho_{0}>0$ such that $\tan (\pi / 2) \rho_{0}=(2 / \alpha) \rho$. Thus we see that $0<$ $\alpha<1.3$ in Theorem 2.1 .

Next, we derive

Theorem 2.2. Let $p(z)$ be analytic in $\mathbf{U}$ with $p(0)=1$ and $p^{\prime}(0)=0$. If $p(z)$ satisfies

$$
\begin{aligned}
& \left|\arg \left(z p^{\prime}(z)+p(z)^{2}+\alpha p(z)\right)\right| \\
& \quad<\frac{\pi}{2} \rho+\operatorname{Tan}^{-1}\left(\frac{2 \rho}{\alpha}\right) \quad(z \in \mathbf{U})
\end{aligned}
$$

for some $\alpha(\alpha>0), \rho\left(\rho_{0} \leqq \rho<1\right)$, where $\rho_{0}(0<$ $\left.\rho_{0}<1\right)$ is given by $\tan (\pi / 2) \rho_{0}=(2 / \alpha) \rho_{0}$, then

$$
|\arg p(z)|<\frac{\pi}{2} \rho \quad(z \in \mathbf{U}) .
$$

Proof. Using the same technique as in the proof of Theorem 2.1, we know that

$$
\operatorname{Tan}^{-1}\left(\frac{2 \rho+a^{\rho} \sin \left(\frac{\pi}{2} \rho\right)}{\alpha+a^{\rho} \cos \left(\frac{\pi}{2} \rho\right)}\right)
$$

is increasing for $a>0$. Thus, we obtain 
$(2.18)$

$$
\begin{gathered}
\left|\arg \left(z_{0} p^{\prime}\left(z_{0}\right)+p\left(z_{0}\right)^{2}+\alpha p\left(z_{0}\right)\right)\right| \\
\quad \geqq \frac{\pi}{2} \rho+\operatorname{Tan}^{-1}\left(\frac{2 \rho}{\alpha}\right)
\end{gathered}
$$

for $z_{0} \in \mathbf{U}$ such that

$$
|\arg p(z)|<\frac{\pi}{2} \rho \quad\left(|z|<\left|z_{0}\right|\right)
$$

and

$$
\left|\arg p\left(z_{0}\right)\right|=\frac{\pi}{2} \rho
$$

This contradicts our condition of the theorem. Therefore,

$$
|\arg p(z)|<\frac{\pi}{2} \rho \quad(z \in \mathbf{U})
$$

Letting $\alpha=1$ in Theorem 2.2, we obtain

Corollary 2.2. Let $p(z)$ be analytic in $\mathbf{U}$ with $p(0)=1$ and $p^{\prime}(0)=0$. If $p(z)$ satisfies

$$
\begin{aligned}
& \left|\arg \left(z p^{\prime}(z)+p(z)^{2}+p(z)\right)\right| \\
& \quad<\frac{\pi}{2} \rho+\operatorname{Tan}^{-1}(2 \rho) \quad(z \in \mathbf{U})
\end{aligned}
$$

for some $\rho(1 / 2 \leqq \rho<1)$, then

$$
|\arg p(z)|<\frac{\pi}{2} \rho \quad(z \in \mathbf{U}) .
$$

Finally, we note that

Remark 2.2. (1) If $\alpha=4 / 5$, then $0<\rho \leqq$ $\rho_{0}$ and $0.647873<\rho_{0}<0.647874$.
(2) If $\alpha=1 / 2$, then $0<\rho \leqq \rho_{0}$ and $0.809251<\rho_{0}<0.809252$.

(3) If $\alpha=1 / 3$, then $0<\rho \leqq \rho_{0}$ and $0.880966<\rho_{0}<0.880967$.

(4) If $\alpha=1 / 4$, then $0<\rho \leqq \rho_{0}$ and $0.913417<\rho_{0}<0.913418$.

(5) If $\alpha=1.1$, then $0<\rho \leqq \rho_{0}$ and $0.401248<$ $\rho_{0}<0.401249$.

(6) If $\alpha=1.2$, then $0<\rho \leqq \rho_{0}$ and $0.262943<$ $\rho_{0}<0.262944$.

(7) If $\alpha=1.3$, then there is no $\rho_{0}>0$ such that $\tan (\pi / 2) \rho_{0}=(2 / \alpha) \rho$. Thus we see that $0<$ $\alpha<1.3$ in Theorem 2.2.

\section{References}

[1] Fukui, S., and Sakaguchi, K.: An extension of a theorem of S. Ruscheweyh. Bull. Fac. Ed. Wakayama Univ. Natur. Sci., 29, 1-3 (1980).

[ 2 ] Hallenbeck, D. J., and Ruscheweyh, S.: Subordinations by convex functions. Proc. Amer. Math. Soc., 52, 191-195 (1975).

[ 3 ] Miller, S. S., and Mocanu, P. T.: Libera transform of functions with bounded turning. J. Math. Anal. Appl., 276, 90-97 (2002).

[ 4 ] Nunokawa, M.: On the order of strongly starlikeness of strongly convex functions. Proc. Japan Acad., 69A, 234-237 (1993). 